\title{
Synthetic properties of bright metal-poor variables
}

\section{BL Hercules stars}

\author{
M. Di Criscienzo ${ }^{1,2}$, F. Caputo ${ }^{3}$, M. Marconi ${ }^{1}$, and S. Cassisi ${ }^{4}$ \\ 1 INAF - Osservatorio Astronomico di Capodimonte, via Moiariello 16, 80131 Napoli, Italy \\ e-mail: marcella@na.astro.it \\ 2 Università degli Studi di Roma "Tor Vergata”, via della Ricerca Scientifica 1, 00133 Roma, Italy \\ 3 INAF - Osservatorio Astronomico di Roma, via Frascati 33, 00040 Monte Porzio Catone, Italy \\ ${ }^{4}$ INAF - Osservatorio Astronomico di Collurania, via Maggini, 64100 Teramo, Italy \\ Received 11 October 2006 / Accepted 16 May 2007
}

\begin{abstract}
We investigate the properties of the so-called BL Her stars, i.e., Population II Cepheids with periods shorter than 8 days, using updated pulsation models and evolutionary tracks computed by adopting a metal abundance in the range of $Z=0.0001$ to $Z=$ 0.004 . We derive the predicted period-magnitude $(P M)$ and period-Wesenheit $(P W)$ relations at the various photometric bands and we show that the slopes of these relations are in good agreement with the slopes determined by observed variables in Galactic globular clusters, independently of the adopted $M_{V}(\mathrm{RR})-[\mathrm{Fe} / \mathrm{H}]$ relation to get the cluster RR Lyrae-based distance. Moreover, we show that the distances provided by the predicted $P M$ and $P W$ relations for BL Her stars agree within the errors with the RR Lyrae-based values. Using the predicted relations with W Vir stars, which are Population II Cepheids with periods longer than 8 days, provides no clear evidence for or against a change in the $P M$ and $P W$ slopes around $P \sim 10$ days.
\end{abstract}

Key words. stars: evolution - stars: population II - stars: variables: general

\section{Introduction}

Population II pulsating variables play a fundamental role in our understanding of the properties of old stellar populations, as well as in the definition of the cosmic distance scale. Among them, RR Lyrae stars are definitively the most abundant and the ones currently used as tracers of dynamical and chemical properties. Moreover, they are used as standard candles to establish the globular cluster distance scale, and they provide the calibration of secondary distance indicators, such as the globular cluster luminosity function in external galaxies (see Di Criscienzo et al. 2006, and references therein).

However, other classes of radial pulsators are actually observed in globular clusters and similar metal-poor stellar fields. In the current nomenclature, they are called Population II Cepheids (P2Cs) and anomalous Cepheids (ACs): the former ones, with periods $P$ from $\sim 1$ to $\sim 25$ days, are observed in clusters with few RR Lyrae stars and blue horizontal branch (HB) morphology, while the latter, with $\sim 0.3 \leq P \leq \sim 2$ days, are observed in the majority of the Local Group dwarf galaxies that have been surveyed for variable stars. These two classes are both brighter but either less massive (P2Cs) or more massive (ACs) than RR Lyrae stars with similar metal content.

In three previous papers (Marconi et al. 2004; Caputo et al. 2004; Fiorentino et al. 2006) dealing with the investigation of the ACs and their role as distance indicators, we discussed the pulsation and evolution properties of these variables and showed that they originate from $Z \leq 0.0004$ central He-burning models more massive than $\sim 1.3 M_{\odot}$, which evolve through the pulsation region at luminosity and effective temperature increasing, on average, as the mass increases. Given the basic equation for radial pulsation $\left(P \rho^{1 / 2}=Q\right.$, where $\rho$ is the star density and $Q$ the pulsation constant), the effect of the higher luminosity is balanced by the higher mass and temperature and consequently, in spite of their bright luminosity, the ACs show periods that are not significantly longer than those typical of RR Lyrae stars.

Concerning the $\mathrm{P} 2 \mathrm{Cs}$, which are often separated ${ }^{1}$ into BL Her stars $(\log P<1)$ and W Vir stars $(\log P>1)$, several authors (e.g., Gingold 1985; Bono et al. 1997; Wallerstein \& Cox 1984; Harris 1985; Wallerstein 1990, 2002) have already suggested that they stem from hot, low-mass stellar structures that started the main central He-burning phase on the blue side of the RR Lyrae gap and now evolve toward the asymptotic giant branch crossing the pulsation region with the luminosity and the effective temperature that increases and decreases, respectively, while decreasing the mass: for this reason, these bright low-mass pulsators should reach periods of several days. Moreover, as also shown by Caputo et al. (2004) (see their Fig. 4) on theoretical grounds, at a fixed period the ACs are more luminous than P2Cs, a feature at the origin of their supposed "anomaly".

On the observational side, Nemec et al. (1994) derived metal-dependent period-luminosity $(P L)$ relations in various photometric bands, suggesting that observed $\mathrm{P} 2 \mathrm{Cs}$ pulsate either in the fundamental or in the first-overtone mode and that the slopes of the $P L$ relations are significantly different for the two modes. On the other hand, on the basis of a sample of P2Cs identified in the OGLE-II variable star catalogue for the Galactic bulge fields, Kubiack \& Udalsky (2003) found that all the observed stars, which have periods from $\sim 0.7$ to about 10 days, follow the same $P L$ relation. Similar results are derived by Pritzl et al. (2003) and Matsunaga et al. (2006) for P2Cs in Galactic

\footnotetext{
1 In their recent reviev, Sandage \& Tammann (2006) adopt a different classification. However, in the present paper we use the classical one.
} 
globular clusters. Furthermore, these last two investigations support the hypothesis that the same $P L$ relation holds for BL Her and W Vir stars, without a steepening of the slope for periods longer than $P \sim 10$ days, as earlier suggested by McNamara (1995).

From the theoretical point of view, the pulsation models by Buchler \& Moskalik (1992) and Buchler \& Buchler (1994), based on a linear and nonlinear radiative analysis, showed that the blue edge for first-overtone pulsation was very close $(\leq 100 \mathrm{~K})$ to the fundamental one, producing a very narrow region of FO-only pulsation. More recently, Bono et al. (1997) computed nonlinear convective models, finding a good agreement between the predicted and the observed boundaries of the P2C instability strip and suggesting that the observed variables are pulsating in the fundamental mode with a typical mass of $\sim 0.52-0.59 M_{\odot}$.

However, the Bono et al. (1997) nonlinear convective models, although also able to provide reliable information on the red edge of pulsation region, were limited to a quite restricted range of stellar parameters and adopted an old input physics (see Bono \& Stellingwerf 1994, for details). For this reason, following our program dealing with a homogeneous study of radially pulsating stars with various chemical compositions, masses, and luminosities, in the present paper we discuss the results of updated pulsation models with mass $0.50-0.65 M_{\odot}$ and luminosity $\log L / L_{\odot}=1.81-2.41$ in order to build a sound theoretical scenario for the analysis of the P2Cs. In particular, we derive the predicted relations connecting evolutionary and pulsation properties for BL Her stars and verify their use as distance indicators.

The paper is organized as follows. In Sect. 2, we present the evolutionary and pulsation models, while in Sect. 3 we deal with the evolution-pulsation connection and give the predicted relations. The comparison with observed variables is presented in Sect. 4 and the conclusions close the paper.

\section{Theoretical framework}

The pulsation models computed for the present paper, as listed in Table 1, adopt the same nonlinear, nonlocal and time-dependent convective hydrodynamical code and the same physical assumptions (i.e., equation of state and opacity tables) already used for the analysis of Classical Cepheids (Caputo et al. 2005; Marconi et al. 2005; Fiorentino et al. 2007), RR Lyrae stars (Marconi et al. 2003; Di Criscienzo et al. 2004) and Anomalous Cepheids (Marconi et al. 2004; Fiorentino et al. 2006). In those papers, several relations connecting pulsational and evolutionary parameters were derived, whose slopes show a general consistency with the observed values. Moreover, they gave also a good agreement with the features of observed light curves of Classical Cepheids (Bono et al. 2002) and RR Lyrae stars (Bono et al. 2000; Castellani et al. 2002; Di Criscienzo et al. 2004; Marconi \& Clementini 2005). For this reason, our pulsation models appear able to provide reliable information on the structural parameters of observed variables and, in turn, on the distance modulus, although we cannot exclude that the theoretical results are affected by unknown systematic errors and that further work is needed to refine our knowledge. However, our computations provide a homogeneous pulsational scenario for the study of complex stellar systems where a variety of pulsating stars can be observed.

The model sequences discussed in this paper are computed as one parameter families with constant chemical composition, mass, and luminosity, by varying the effective temperature $T_{\mathrm{e}}$ by steps of $100 \mathrm{~K}$. These models, which adopt a value of the
Table 1. Basic parameters of the pulsation models and resulting effective temperatures at the edges for fundamental and first-overtone pulsation.

\begin{tabular}{lcccccc}
\hline \hline$Z$ & $M$ & $\log L$ & $\mathrm{FOBE}^{a}$ & $\mathrm{FBE}^{b}$ & $\mathrm{FORE}^{c}$ & $\mathrm{FRE}^{d}$ \\
\hline 0.0001 & 0.60 & 1.95 & - & 6850 & - & 5750 \\
& & 2.05 & - & 6750 & - & 5650 \\
& & 2.15 & - & 6750 & - & 5550 \\
& 0.65 & 1.91 & 6950 & 6850 & 6050 & 5750 \\
& & 2.01 & 6750 & 6850 & 6250 & 5750 \\
& & 2.11 & - & 6750 & - & 5550 \\
\hline 0.001 & 0.50 & 2.11 & - & 6650 & - & 5450 \\
& 0.50 & 2.41 & - & 6350 & - & 5150 \\
& 0.55 & 1.81 & 6850 & 6850 & 6350 & 5650 \\
& & 1.91 & - & 6850 & - & 5550 \\
& & 2.01 & - & 6750 & - & 5450 \\
& 0.65 & 1.81 & 7050 & 6750 & 6650 & 5750 \\
& & 1.91 & 6850 & 6750 & 6150 & 5650 \\
0.004 & 0.55 & 1.81 & - & 6950 & - & 5750 \\
& & 1.91 & - & 6850 & - & 5650 \\
& & 2.01 & - & 6750 & - & 5450 \\
\hline
\end{tabular}

Note: ${ }^{a}$ First Overtone Blue Edge; ${ }^{b}$ Fundamental Blue Edge; ${ }^{c}$ First Overtone Red Edge; ${ }^{d}$ Fundamental Red Edge. If no value is given at FOBE and FORE, we found only fundamental models. A helium abundance $Y=0.24$ and a mixing-length parameter $l / H_{\mathrm{p}}=1.5$ has been adopted. Mass and luminosity are in solar units.

mixing length parameter $l / H_{\mathrm{p}}=1.5$ to close the system of convective and dynamic equations, are fully described by Marconi \& Di Criscienzo (2007), so we report only the results relevant for the purpose of the present paper. For the sake of the following discussion, let us first make clear that increasing (decreasing) by $100 \mathrm{~K}$ the effective temperature of the computed bluest (reddest) fundamental (F) or first-overtone (FO) model yields nonpulsating structures in the corresponding mode. Accordingly, we adopt the effective temperature of the computed bluest FO and $\mathrm{F}$ models, increased by $50 \mathrm{~K}$, as the first-overtone (FOBE) and the fundamental blue edge (FBE), respectively, and the effective temperature of the reddest FO and F models, decreased by $50 \mathrm{~K}$, as the first-overtone (FORE) and the fundamental red edge (FRE), respectively. This yields that the effective temperatures given in Table 1 have the intrinsic uncertainty of $\pm 50 \mathrm{~K}$.

Starting with the models with $0.65 M_{\odot}$ and $\log L / L_{\odot}=1.81$, we note that they follow the well-known behavior of RR Lyrae stars in that FO models are generally bluer than the F ones, but with the FORE redder than the FBE. As a consequence, we find that: $a$ ) the limits of the whole pulsation region are described by the FOBE and the FRE; $b$ ) both the pulsation modes are stable in the middle zone delimited by the FBE and the FORE; c) F-only pulsators are located between the FRE and the FORE and FO-only pulsators between the FBE and the FOBE. By increasing the luminosity, the whole pulsation region moves towards the red, but with a significant shrinking of the FO-only pulsation region. Based on the values listed in Table 1, the difference FOBE-FBE is $\sim+300 \mathrm{~K}$ at $\log L / L_{\odot}=1.81$ and $\sim+100 \mathrm{~K}$ at $\log L / L_{\odot}=1.91$. A further increase of the luminosity yields that the FOBE becomes redder than the FBE (with a difference FOBE-FBE $\sim-150 \mathrm{~K}$ at $\log L / L_{\odot}=2.01$ ), with the total disappearance of stable FO models at $\log L / L_{\odot} \geq 2.11$.

When varying the mass, we note that no FO model with $0.60 M_{\odot}$ is stable at $\log L / L_{\odot} \geq 1.95$, while with $0.55 M_{\odot}$ we get $\mathrm{FOBE}=\mathrm{FBE}$ at $\log L / L_{\odot}=1.80$ and only $\mathrm{F}$ models above this luminosity level. On the other hand, by relying on the 
computations discussed by Marconi et al. (2003) and Di Criscienzo et al. (2004), we recall that, for models with $0.80 M_{\odot}$, the difference FOBE-FBE is $\sim+400 \mathrm{~K}$ at $\log L / L_{\odot}=$ 1.72 and $\sim+200 \mathrm{~K}$ at $\log L / L_{\odot}=1.91$.

In summary, the results listed in Table 1 confirm earlier suggestions (see Tuggle \& Iben 1972; Bono et al. 1995, 1997) that an "intersection" luminosity $L_{\mathrm{IP}}$ exists, for each given mass and helium content, where FOBE $=$ FBE, and that only the fundamental mode is stable above this luminosity. On these grounds, one finds that the red limit of the instability strip is always determined by the FRE, while the blue limit is given by the FOBE or the FBE depending on whether the luminosity is fainter or brighter, respectively, than $L_{\mathrm{IP}}$.

Based on present computations and the quoted RR Lyrae models, we estimate at $Y=0.24$

$\log L_{\mathrm{IP}} \sim 2.3+1.9 \log M$,

where mass and luminosity are in solar units. Concerning the limits of the instability strip, we adopt

$\log T_{\mathrm{e}}(\mathrm{FOBE})=3.970( \pm 0.004)-0.057 \log L+0.094 \log M,(2)$

as determined by Marconi et al. (2003) from pulsation models with $L<L_{\mathrm{IP}}$, while a linear interpolation through the present results gives

$$
\begin{aligned}
& \log T_{\mathrm{e}}(\mathrm{FBE})=3.912( \pm 0.007)-0.035 \log L+0.048 \log M \\
& \log T_{\mathrm{e}}(\mathrm{FRE})=3.925( \pm 0.006)-0.075 \log L+0.118 \log M,
\end{aligned}
$$

where the uncertainties include the intrinsic uncertainty of $\pm 50 \mathrm{~K}$ on the FOBE, FBE, and FRE temperatures. Moreover, we derive that the pulsation equation for the fundamental mode can be approximated as

$$
\begin{aligned}
\log P_{\mathrm{F}}= & 11.579( \pm 0.015)+0.89 \log L \\
& -0.89 \log M-3.54 \log T_{\mathrm{e}}
\end{aligned}
$$

while for the few first-overtone models we get $\log P_{\mathrm{FO}} \sim \log P_{\mathrm{F}}-$ 0.12 , at constant mass, luminosity, and effective temperature. Before proceeding, it is worth mentioning that the adopted helium content $Y=0.24$ accounts for the most recent estimate (Cassisi et al. 2003; Salaris et al. 2004) based on measurements of the $R$ parameter ${ }^{2}$ in a large sample of Galactic globular clusters. To quantify the dependence on the helium abundance, we computed specific models for RR Lyrae with $Y=0.20$ and 0.35 showing that reasonable variations in the helium content $(\Delta Y= \pm 0.03)$ in metal-poor stars have negligible effects on the effective temperature of the instability strip edges $\left(\Delta T_{\mathrm{e}} \sim\right.$ $\pm 80 \mathrm{~K}$ ). Moreover, we wish to recall that the onset of pulsation also depends on the efficiency of convection in the star's external layers, namely on the adopted value of the mixing length parameter $l / H_{\mathrm{p}}$. Since the effect of convection is to quench pulsation and the depth of convection increases from high to low effective temperatures, we expect that varying the $l / H_{\mathrm{p}}$ value will modify the effective temperature at FRE by a larger amount with respect to FBE or FOBE. Indeed, the additional computations with $l / H_{\mathrm{p}}=2.0$ computed by Marconi \& Di Criscienzo (2007) have confirmed the general trend shown by RR Lyrae (Marconi et al. 2003; Di Criscenzo et al. 2004) and classical Cepheid models (Fiorentino et al. 2007) in that, at constant mass and luminosity, the FBE and FRE effective temperatures increase by $\sim 100$ and $\sim 300 \mathrm{~K}$, respectively. The effects of different $l / H_{\mathrm{p}}$ values on the predicted relations will be discussed in the next section.

\footnotetext{
2 This parameter is the number ratio of $\mathrm{HB}$ to red giant branch stars brighter than the HB level.
}

Table 2. Chemical compositions of the adopted evolutionary models.

\begin{tabular}{lccc}
\hline \hline$Z$ & $Y$ & {$[\alpha / \mathrm{Fe}]$} & {$[\mathrm{Fe} / \mathrm{H}]$} \\
\hline 0.0001 & 0.245 & 0.4 & -2.62 \\
0.001 & 0.246 & 0.4 & -1.62 \\
0.004 & 0.251 & 0.4 & -1.01 \\
0.0001 & 0.245 & 0.0 & -2.27 \\
0.001 & 0.246 & 0.0 & -1.27 \\
0.004 & 0.251 & 0.0 & -0.66 \\
\hline
\end{tabular}

For the evolutionary framework, we adopt the models computed by Pietrinferni et al. $(2004,2006)$ for scaled solar and $\alpha$-enhanced $([\alpha / \mathrm{Fe}]=0.4)$ metal distributions in order to cover (see Table 2) the $[\mathrm{Fe} / \mathrm{H}]$ range between -2.6 and -0.7 . All the models have been transferred from the theoretical HR diagram to the various observational planes by adopting updated coloreffective temperature relations and bolometric corrections (see Pietrinferni et al. 2004; Cassisi et al. 2004) and the reader is referred to these papers ${ }^{3}$ for information on the physical inputs and numerical assumptions. Here, it seems sufficient to note that this evolutionary framework is based on the most updated physical scenario and that the various stellar models have been followed all through the main core H-burning phase and advanced core and shell He-burning evolutionary phases. All the He-burning models adopted in the present analysis were computed by accounting for an He-core mass and He-envelope abundance on the zero age horizontal branch (ZAHB) characteristic of a red giant branch (RGB) progenitor with an initial total mass equal to $\sim 0.8 M_{\odot}$, corresponding to an age at the RGB tip on the order of 13 Gyr. The reliability and accuracy of the whole evolutionary scenario have already been tested by comparison with various empirical data sets (see also Riello et al. 2003; Salaris et al. 2004; Recio-Blanco et al. 2005) and, in summary, it appears quite suitable for investigating the populations of variable stars in Galactic globular clusters. Finally, let us note that these evolutionary computations so far represent the most updated and complete set of low-mass, He-burning models currently available. As a fact, the unique set of similar stellar models is the one published a long time ago by Dorman et al. (1993), which is based on physical inputs that are any longer updated. However, in the following section we discuss how the uncertainties in the evolutionary framework would affect the predicted relations.

The procedure for deriving the observational parameters of the predicted pulsators is in principle quite simple and has been described in several previous investigations (Bono et al. 1997; Marconi et al. 2003; Fiorentino et al. 2006). As shown in Fig. 1, the relations of the predicted edges of the instability strip (Eqs. (2)-(4)) give us the way to select the models evolving with a luminosity higher than $\log L_{\mathrm{IP}}$ and showing $\mathrm{FBE} \geq \log T_{\mathrm{e}} \geq$ FRE. In this way, we find that the mass range of the predicted fundamental pulsators varies from $0.515-0.62 M_{\odot}$ at $[\mathrm{Fe} / \mathrm{H}]=$ -2.6 to $0.50-0.55 M_{\odot}$ at $[\mathrm{Fe} / \mathrm{H}]=-0.7$. These mass values are coherent with the mass range of the pulsating models listed in Table 1 . However, as shown in Table 3 , the average luminosity of the pulsators with a given mass increases as the metal content decreases, yielding that the $[\mathrm{Fe} / \mathrm{H}]=-2.6$ pulsators less massive than $0.53 M_{\odot}$ are more luminous than our brightest pulsating models. Since we cannot a priori be sure that the edge and period relations provided by the pulsation models listed in Table 1 can be extrapolated to higher luminosity levels, in the following we will use only the predicted pulsators whose mass and luminosity

3 The whole set of stellar models can be retrieved at the following URL site: http://www . te . astro.it/BASTI/index . php 


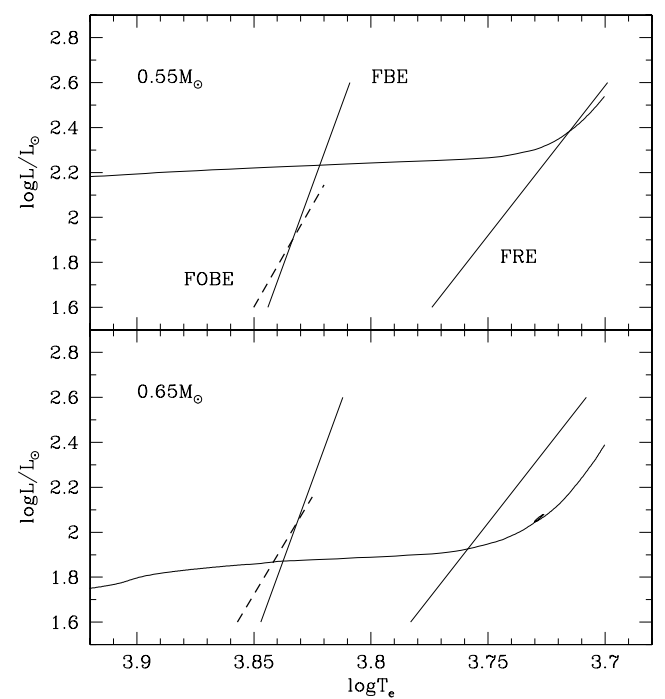

Fig. 1. Selected evolutionary tracks with $Z=0.0001$ and $[\alpha / \mathrm{Fe}]=0.4$ in comparison with the predicted FOBE, FBE and FRE. At luminosity levels brighter than the intersection between FOBE and FBE, we found only fundamental models.

Table 3. Luminosity $L_{\mathrm{FBE}}$ at the fundamental blue edge of the models with the labeled metal content and $[\alpha / \mathrm{Fe}]=0.4$, compared to the luminosity $L_{\mathrm{IP}}$ at the intersection between FOBE and FBE.

\begin{tabular}{ccccc}
\hline \hline$M / M_{\odot}$ & $\log L_{\mathrm{FBE}}$ & $\log L_{\mathrm{FBE}}$ & $\log L_{\mathrm{FBE}}$ & $\log L_{\mathrm{IP}}$ \\
& $Z=0.0001$ & $Z=0.001$ & $Z=0.004$ & \\
\hline 0.500 & - & - & 2.62 & 1.73 \\
0.505 & - & - & $\mathbf{2 . 3 9}$ & 1.74 \\
0.510 & - & 2.55 & $\mathbf{2 . 2 8}$ & 1.74 \\
0.515 & 3.08 & 2.43 & $\mathbf{2 . 2 0}$ & 1.75 \\
0.520 & 2.79 & $\mathbf{2 . 3 2}$ & $\mathbf{2 . 1 5}$ & 1.76 \\
0.525 & 2.55 & $\mathbf{2 . 2 5}$ & $\mathbf{2 . 0 8}$ & 1.77 \\
0.530 & 2.46 & $\mathbf{2 . 2 1}$ & $\mathbf{2 . 0 2}$ & 1.78 \\
0.535 & $\mathbf{2 . 3 8}$ & $\mathbf{2 . 1 6}$ & $\mathbf{1 . 9 9}$ & 1.78 \\
0.540 & $\mathbf{2 . 3 2}$ & $\mathbf{2 . 1 1}$ & $\mathbf{1 . 9 4}$ & 1.79 \\
0.545 & $\mathbf{2 . 2 7}$ & $\mathbf{2 . 0 7}$ & $\mathbf{1 . 8 9}$ & 1.80 \\
0.550 & $\mathbf{2 . 2 4}$ & $\mathbf{2 . 0 4}$ & $\mathbf{1 . 8 3}$ & 1.81 \\
0.560 & $\mathbf{2 . 1 6}$ & $\mathbf{1 . 9 8}$ & - & 1.82 \\
0.570 & $\mathbf{2 . 1 0}$ & $\mathbf{1 . 9 5}$ & - & 1.84 \\
0.580 & $\mathbf{2 . 0 5}$ & $\mathbf{1 . 8 8}$ & - & 1.85 \\
0.590 & $\mathbf{2 . 0 2}$ & $\mathbf{1 . 8 7}$ & - & 1.86 \\
0.600 & $\mathbf{2 . 0 1}$ & - & - & 1.88 \\
0.610 & $\mathbf{1 . 9 6}$ & - & - & 1.89 \\
0.620 & $\mathbf{1 . 9 3}$ & - & - & 1.91 \\
\hline
\end{tabular}

Note: the models in bold face are among those adopted for deriving the predicted relations (see text). The luminosity values are in solar units.

are consistent with those adopted for the pulsation models, as given in bold face in Table 3 .

\section{The connection between stellar evolution and pulsation}

By calculating the fundamental period by means of Eq. (5) and adopting the magnitudes computed by Pietrinferni et al. (2004, 2006), we show in Fig. 2 selected Period-Magnitude $(P M)$ diagrams of the predicted fundamental pulsators with $l / H_{\mathrm{p}}=1.5$, $Z=0.0001$ and $[\alpha / \mathrm{Fe}]=0.4$. Note that the resulting periods are in the range of about 0.8 to 8 days, making our theoretical investigations quite appropriate for analyzing of observed BL Her stars.

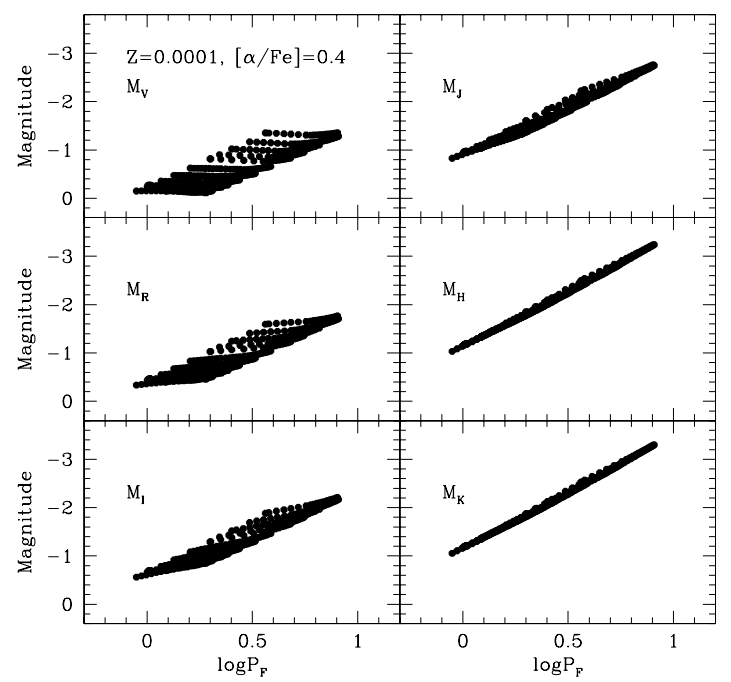

Fig. 2. Period-magnitude diagrams of fundamental pulsators with $l / H_{\mathrm{p}}=1.5, Z=0.0001$ and $[\alpha / \mathrm{Fe}]=0.4$.

Table 4. Predicted $P M_{I}, P M_{J}, P M_{H}$, and $P M_{K}$ relations for fundamental pulsators with iron content in the range of $[\mathrm{Fe} / \mathrm{H}]=-2.6$ to -0.7 and $P \leq 8$ days.

\begin{tabular}{ccccc}
\hline \hline \multicolumn{5}{c}{$M_{i}=a+b \log P_{\mathrm{F}}+c[\mathrm{Fe} / \mathrm{H}]+d\left(l / H_{\mathrm{p}}-1.5\right)$} \\
\hline$M_{i}$ & $a$ & $b$ & $c$ & $d$ \\
\hline$M_{I}$ & $-0.26 \pm 0.19$ & $-2.10 \pm 0.06$ & $+0.04 \pm 0.01$ & -0.24 \\
$M_{J}$ & $-0.64 \pm 0.13$ & $-2.29 \pm 0.04$ & $+0.04 \pm 0.01$ & -0.16 \\
$M_{H}$ & $-0.95 \pm 0.06$ & $-2.34 \pm 0.02$ & $+0.06 \pm 0.01$ & -0.08 \\
$M_{K}$ & $-0.97 \pm 0.06$ & $-2.38 \pm 0.02$ & $+0.06 \pm 0.01$ & -0.06 \\
\hline
\end{tabular}

As already found for other pulsating variables, the effect of the intrinsic width in effective temperature of the instability strip (see Fig. 1) is greatly reduced when moving from optical to near-infrared magnitudes. On this basis, it is quite clear that synthetic $P M_{B}$ to $P M_{R}$ relations will significantly depend on the actual distribution of the pulsators within the pulsation region, at variance with the case of the near-infrared magnitudes. Moreover, we wish to recall that a variation in the mixing length parameter from $l / H_{\mathrm{p}}=1.5$ to 2.0 gives hotter FBE and FRE by about $100 \mathrm{~K}$ and $300 \mathrm{~K}$, respectively. Consequently, the pulsator distribution is slightly shifted toward shorter periods, yielding mildly steepened (less than $2 \%$ ) and brighter $P M$ relations, mainly in the optical bands. A least-square fit to all the fundamental models yields the linear relations listed in Table 4: as a result, we find that the apparent distance modulus $\mu_{I}$ of observed variables can be determined within $\pm 0.20 \mathrm{mag}$, including the uncertainty due to the mixing-length parameter, whereas both $\mu_{H}$ and $\mu_{K}$ can be determined with a formal accuracy of 0.07 mag. Given the residual effect of the intrinsic width of the instability strip, the $P M_{J}$ relation yields $\mu_{J}$ within $0.15 \mathrm{mag}$.

It is widely acknowledged that the scatter in optical magnitudes can be removed if a period-magnitude-color $(P M C)$ is considered, i.e., if the pulsator magnitude is given as a function of the period and color. Several previous papers (see, e.g., Madore 1982; Madore \& Freedman 1991; Tanvir 1999; Caputo et al. 2000, 2004) have already shown that the color coefficient of the various $P M C$ relations is not too different from the extinction-toreddening ratio provided by optical and near-infrared reddening laws (see Dean et al. 1978; Caldwell \& Coulson 1987; Cardelli et al. 1989; Laney \& Stobie 1993). On this basis, adopting the reddening insensitive Wesenheit functions, where the magnitude 


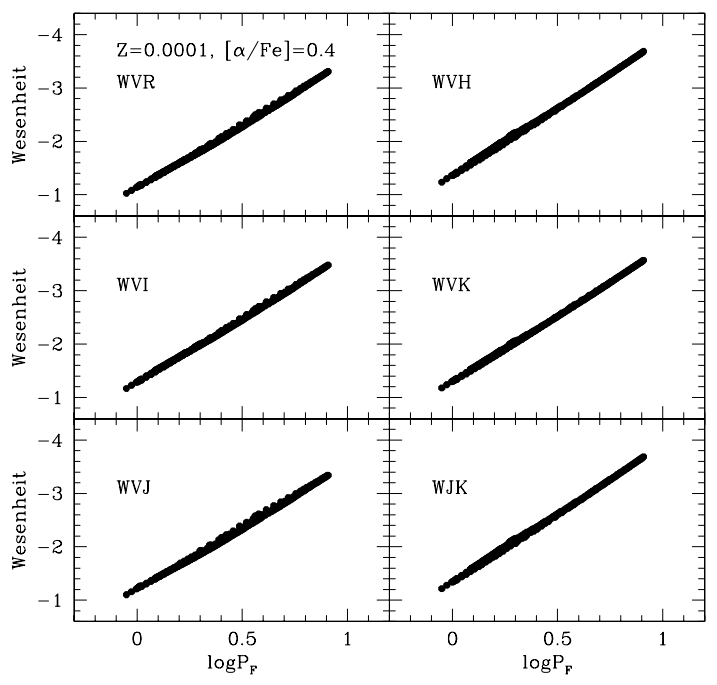

Fig. 3. Selected period-Wesenheit diagrams of fundamental pulsators with $l / H_{\mathrm{p}}=1.5, Z=0.0001$ and $[\alpha / \mathrm{Fe}]=0.4$.

Table 5. PW relations for fundamental pulsators with iron content in the range of $[\mathrm{Fe} / \mathrm{H}]=-2.6$ to -0.7 and period $P \leq 8$ days.

\begin{tabular}{lcccc}
\hline \hline \multicolumn{5}{c}{$W=a+b \log P_{\mathrm{F}}+c[\mathrm{Fe} / \mathrm{H}]+d\left(l / H_{\mathrm{p}}-1.5\right)$} \\
\hline$W$ & $a$ & $b$ & $c$ & $d$ \\
\hline$W V R$ & $-1.07 \pm 0.07$ & $-2.42 \pm 0.02$ & $+0.01 \pm 0.01$ & -0.10 \\
$W V I$ & $-1.16 \pm 0.07$ & $-2.43 \pm 0.02$ & $+0.04 \pm 0.01$ & -0.10 \\
$W V J$ & $-1.04 \pm 0.06$ & $-2.37 \pm 0.02$ & $+0.05 \pm 0.01$ & -0.08 \\
$W V H$ & $-1.20 \pm 0.06$ & $-2.58 \pm 0.02$ & $+0.06 \pm 0.01$ & -0.07 \\
$W V K$ & $-1.13 \pm 0.06$ & $-2.52 \pm 0.02$ & $+0.06 \pm 0.01$ & -0.06 \\
$W J K$ & $-1.15 \pm 0.06$ & $-2.60 \pm 0.02$ & $+0.06 \pm 0.01$ & -0.06 \\
\hline \multicolumn{4}{c}{}
\end{tabular}

is corrected for the color according to the interstellar extinction, also removes the largest part of the effect of differing effective temperatures. In the following, when adopting $A_{V}=3.1 E(B-V)$, $A_{R}=2.45 E(B-V), A_{I}=1.85 E(B-V), A_{J}=0.897 E(B-V)$, $A_{H}=0.574 E(B-V)$ and $A_{K}=0.372 E(B-V)$, we will refer to the Wesenheit functions $W B V=V-3.1 E(B-V), W V R=$ $V-4.77(V-R), W V I=V-2.48(V-I), W V J=V-1.407(V-J)$, $W V H=V-1.227(V-H)$, and $W V K=V-1.136(V-K)$. Moreover, since only near-infrared data are available for several $\mathrm{P} 2 \mathrm{Cs}$, we will consider also the function $W J K=K-0.709(J-K)$.

As shown in Fig. 3, where the fundamental pulsators with $l / H_{\mathrm{p}}=1.5, Z=0.0001$, and $[\alpha / \mathrm{Fe}]=0.4$ are plotted, in some selected PW diagrams, the magnitude dispersion at fixed period is indeed greatly reduced, leading to tight linear $P W$ relations. With regard to the effect of an increased value of the mixing length parameter, we find that moving from $l / H_{\mathrm{p}}=1.5$ to 2.0 yields slightly brighter $P W$ relations, while leaving the slope almost unvaried (less than 2\%). By a least square fit to all the fundamental pulsators, we derive the coefficients listed in Table 5. These relations give us a very safe way to estimate the intrinsic distance modulus $\mu_{0}$ of observed variables with a formal accuracy of $\sim 0.1 \mathrm{mag}$, independent of the reddening. Concerning the $W B V$ function, we show in Fig. 4 that the pulsator distribution in the $\log P_{\mathrm{F}}-W B V$ plane is much better represented by a quadratic relation, i.e., $W B V=\alpha+\beta \log P_{\mathrm{F}}+\gamma\left(\log P_{\mathrm{F}}\right)^{2}$, mainly for the lower metal content. Note also that, at variance with the other Wesenheit functions, the $W B V$ function becomes brighter as the pulsator metal content increases, at a fixed period, with the magnitude difference increasing towards the longer periods. As a whole, the least-square fit to all the fundamental pulsators

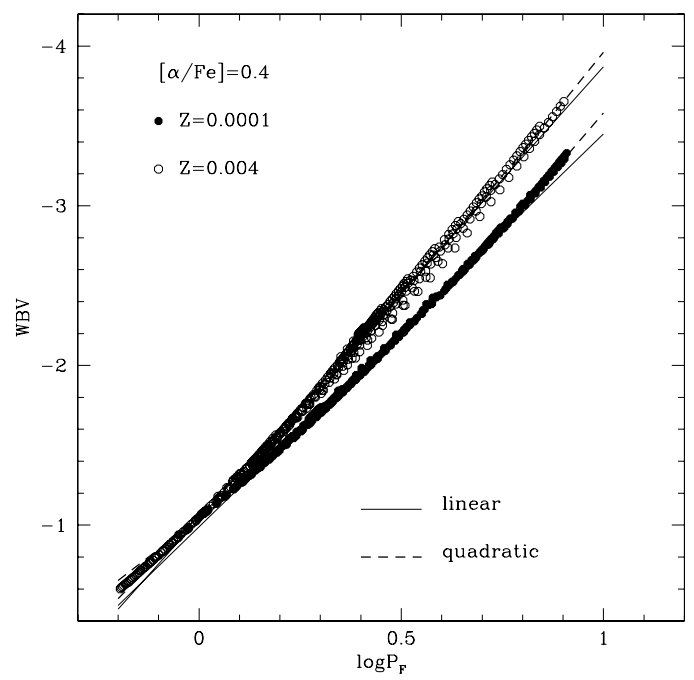

Fig. 4. $W B V$ function versus period of selected fundamental pulsators with $l / H_{\mathrm{p}}=1.5$. The solid and dashed lines are the linear and quadratic fits, respectively.

yields $\alpha=-1.06( \pm 0.09), \beta=-2.96( \pm 0.08)-0.36[\mathrm{Fe} / \mathrm{H}]$ and $\gamma=-0.17( \pm 0.05)+0.13[\mathrm{Fe} / \mathrm{H}]$. To close of this section, let us note that the dependence of the HB luminosity at the RR Lyrae gap on the metal content $Z$ seems to be a robust result of stellar evolution and that all the available sets of evolutionary models, with few exceptions, predict similar trends (see Fig. 13 in Pietrinferni et al. 2004). Unfortunately, no comparison with other recent models can be made for He-burning low-mass models, so we adopt an uncertainty of about \pm 0.04 dex of the logarithm luminosity as a safe estimate. However, when accounting for the dependence of the pulsation period on the stellar luminosity, this uncertainty on the stellar brightness has no significative effects on the predicted PM and PW relations given in Tables 4 and 5 , respectively. Indeed, an increase of 0.04 dex in the luminosity, for any fixed effective temperature, causes a period variation $\delta \log P=0.036$, while all the magnitudes and Wesenheit functions become brighter by $0.1 \mathrm{mag}$. As a consequence of these simultaneous variations, the "new" $P M$ and $P W$ relations will be brighter by 0.02 mag at most.

\section{Comparison with observations}

The Galactic globular clusters with observed P2Cs are listed in Table 6 with their reddening $E(B-V)$, apparent visual magnitude $V(\mathrm{HB})$, and HB type, as given by Harris (1996). We recall that the HB type is the ratio $(B-R) /(B+V+R)$, where $V$ is the number of RR Lyrae variables, while $B$ and $R$ are the numbers of HB stars that are bluer and redder, respectively, than RR Lyrae stars. For all the P2Cs, we adopt the periods and the apparent magnitudes provided by Pritzl et al. (2003, BVI, hereafter Pr03) and Matsunaga et al. (2006, $J H K_{\mathrm{s}}$, hereafter Ma06).

In their investigations, Pr03 and Ma06 find fairly tight linear correlations of the absolute magnitudes of P2Cs, as derived from RR Lyrae-based distance moduli, with $\log P$, without clear evidence of a change in the slope between BL Her and W Vir stars. In particular, by adopting $M_{V}(\mathrm{RR})=0.89+$ $0.22[\mathrm{Fe} / \mathrm{H}], \mathrm{Ma} 06$ derive $\delta M_{J} / \delta \log P=-2.23( \pm 0.05), \delta M_{H} / \delta$ $\log P=-2.34( \pm 0.05)$ and $\delta M_{K_{\mathrm{s}}} / \delta \log P=-2.41( \pm 0.05)$, which are in excellent agreement with the predicted slopes given in Table 4. In that study, it is also mentioned that the slope of the observed near-infrared $P M$ relations is not affected by 
Table 6. Galactic globular clusters with observed P2Cs.

\begin{tabular}{|c|c|c|c|c|c|c|}
\hline Name & $E(B-V)$ & {$[\mathrm{Fe} / \mathrm{H}]$} & $V(\mathrm{HB})$ & HB type & $N_{\mathrm{BL}}$ & $N_{\mathrm{WV}}$ \\
\hline HP1 & 1.19 & -1.55 & 18.60 & - & 0 & 2 \\
\hline N1904 & 0.01 & -1.57 & 16.15 & +0.89 & 0 & 1 \\
\hline N2419 & 0.11 & -2.12 & 20.45 & +0.86 & 1 & 0 \\
\hline N2808 & 0.22 & -1.15 & 16.22 & -0.49 & 1 & 0 \\
\hline N4372 & 0.39 & -2.09 & 15.50 & +1.00 & 2 & 0 \\
\hline N5139- $\omega$ Cen & 0.12 & -1.62 & 14.53 & +0.94 & 9 & 2 \\
\hline N5272-M3 & 0.01 & -1.57 & 15.68 & +0.08 & 0 & 1 \\
\hline N5904-M5 & 0.03 & -1.27 & 15.07 & +0.31 & 0 & 2 \\
\hline N5986 & 0.28 & -1.58 & 16.52 & +0.97 & 0 & 1 \\
\hline N6093-M80 & 0.18 & -1.75 & 16.10 & +0.93 & 0 & 1 \\
\hline N6205-M13 & 0.02 & -1.54 & 15.05 & +0.97 & 4 & 1 \\
\hline N6218-M12 & 0.19 & -1.48 & 14.60 & +0.97 & 0 & 1 \\
\hline N6229 & 0.01 & -1.43 & 18.03 & +0.24 & 0 & 1 \\
\hline N6254-M10 & 0.28 & -1.52 & 14.65 & +0.98 & 1 & 2 \\
\hline N6256 & 1.03 & -0.70 & 18.50 & - & 0 & 1 \\
\hline N6266-M62 & 0.47 & -1.29 & 16.25 & +0.32 & 0 & 1 \\
\hline N6273-M19 & 0.41 & -1.68 & 16.50 & - & 1 & 2 \\
\hline N6284 & 0.28 & -1.32 & 17.40 & - & 2 & 0 \\
\hline N6293 & 0.41 & -1.92 & 16.50 & +0.90 & 1 & 0 \\
\hline N6325 & 0.89 & -1.17 & 17.90 & - & 0 & 2 \\
\hline N6341-M92 & 0.02 & -2.28 & 15.10 & +0.91 & 1 & 0 \\
\hline N6388 & 0.37 & -0.60 & 16.85 & - & 2 & 1 \\
\hline N6402-M14 & 0.60 & -1.39 & 17.30 & +0.65 & 2 & 3 \\
\hline N6441 & 0.47 & -0.53 & 17.51 & - & 2 & 5 \\
\hline N6453 & 0.66 & -1.53 & 17.53 & - & 0 & 2 \\
\hline N6569 & 0.55 & -0.86 & 17.52 & - & 0 & 1 \\
\hline N6626-M28 & 0.40 & -1.45 & 15.55 & +0.90 & 0 & 2 \\
\hline N6626-M28 & 0.40 & -1.45 & 15.55 & +0.90 & 0 & 1 \\
\hline N6715-M54 & 0.15 & -1.58 & 18.17 & +0.75 & 2 & 0 \\
\hline N6749 & 1.50 & -1.60 & 19.70 & +1.00 & 1 & 0 \\
\hline N6752 & 0.04 & -1.56 & 13.70 & +1.00 & 1 & 0 \\
\hline N6779-M56 & 0.20 & -1.94 & 16.16 & +1.00 & 1 & 1 \\
\hline N7078-M15 & 0.10 & -2.26 & 15.83 & +0.67 & 2 & 1 \\
\hline N7089-M2 & 0.06 & -1.62 & 16.05 & +0.96 & 0 & 4 \\
\hline Ton1 & 2.28 & -1.30 & 21.40 & - & 0 & 1 \\
\hline
\end{tabular}

different assumptions on the slope of the $M_{V}(\mathrm{RR})-[\mathrm{Fe} / \mathrm{H}]$ relation. In fact, by repeating the $\mathrm{Ma} 06$ procedure but adopting $M_{V}(\mathrm{RR})=0.8 \mathrm{mag}$ and $M_{V}(\mathrm{RR})=0.94+0.30[\mathrm{Fe} / \mathrm{H}]$ (Sandage 1993: S93), we show in Fig. 5 (upper panels) that the variation to the near-infrared $P M$ slopes is less than 2 percent. Here, we add that this holds also for the $P W$ relations based on $V J H K_{\mathrm{s}}$ magnitudes (lower panels). The arrow in this figure refers to NGC 6341 V7 (Del Principe et al. 2005) which will be discussed separately.

Turning to the Pr03 optical magnitudes, some comments should be made to the data plotted in Fig. 6. First, even removing the too faint outliers (i.e., the BVI data of V24 and V28 in NGC 4372 and the $B V$ data of V12 and V32 in NGC 6205 and of V3 in NGC 6254, see also Pr03), a significant dispersion in the $P M_{V}$ and $P W B V$ planes, as well as some evidence that the W Vir stars follow steeper $P M_{V}$ relations than the BL Her stars can be noticed. The observed $P M_{I}$ and $P W V I$ relations appear linear and quite tight, with the slope independent of the adopted $M_{V}(\mathrm{RR})-[\mathrm{Fe} / \mathrm{H}]$ relation and in good agreement with the predicted values. However, it should be mentioned that these results are based on a rather small number of data points (mostly, the variables in NGC 6441).

In summary, we derive that all the observed P2Cs show linear near-infrared $P M$ relations and linear $P W$ relations, with the exclusion of $W B V$, independently of the adopted $M_{V}(\mathrm{RR})-[\mathrm{Fe} / \mathrm{H}]$ relation. Moreover, the slopes of these relations are in close agreement with our predicted values for variables with $P \leq 8$ days, supporting the hypothesis of similar relations for BL Her and W Vir stars. In addition, we wish to mention that the $[\mathrm{Fe} / \mathrm{H}]$ effect on the zero-point of the near-infrared $P M$ relations, as estimated by Ma06, is about $0.1 \mathrm{mag} / \mathrm{dex}^{-1}$, again in agreement with our theoretical value.

On this ground, we can use the predicted relations derived in the previous section to derive the $\mathrm{P} 2 \mathrm{C}$ distance moduli. With this purpose, since the Pietrinferni et al. $(2004,2006)$ magnitudes are in the Bessell \& Brett (1988) near-infrared photometric system, the relations provided by Carpenter (2001) are used to transform the original 2MASS $J H K_{\mathrm{s}}$ data given by Ma06 into standard $J H K$ magnitudes. For the $\mathrm{P} 2 \mathrm{C}$ reddening and metal content, we adopt the values of the hosting globular cluster; however, for NGC 6388 and NGC 6441 we consider also $[\mathrm{Fe} / \mathrm{H}]=-2.0$, as adopted by $\mathrm{PrO3}$ and $\mathrm{Ma06}$.

By excluding the globular clusters with only W Vir stars and NGC 6341 V7, which will be discussed separately, we find some points worthy of mention:

- from the BVI magnitudes of the NGC $6441 \mathrm{P} 2 \mathrm{Cs}$, we find an increasing discrepancy between the $\mu_{0, W B V}$ and $\mu_{0, W V I}$ values of a given variable when moving from short to long period stars. With $[\mathrm{Fe} / \mathrm{H}]=-0.53$, we get $\mu_{0, W B V}-\mu_{0, W V I} \sim 0.4 \mathrm{mag}$ at $\log P \sim 1.1$ and $\sim 0.8$ mag at $\log P \sim 1$.3. It is interesting to note that, if $[\mathrm{Fe} / \mathrm{H}]=-2.0$ is adopted, these differences are reduced to $\sim 0$ and $\sim 0.3 \mathrm{mag}$, respectively. This would suggest that, despite the cluster high metallicity, the P2Cs may have a low metal content. However, we remind that the 


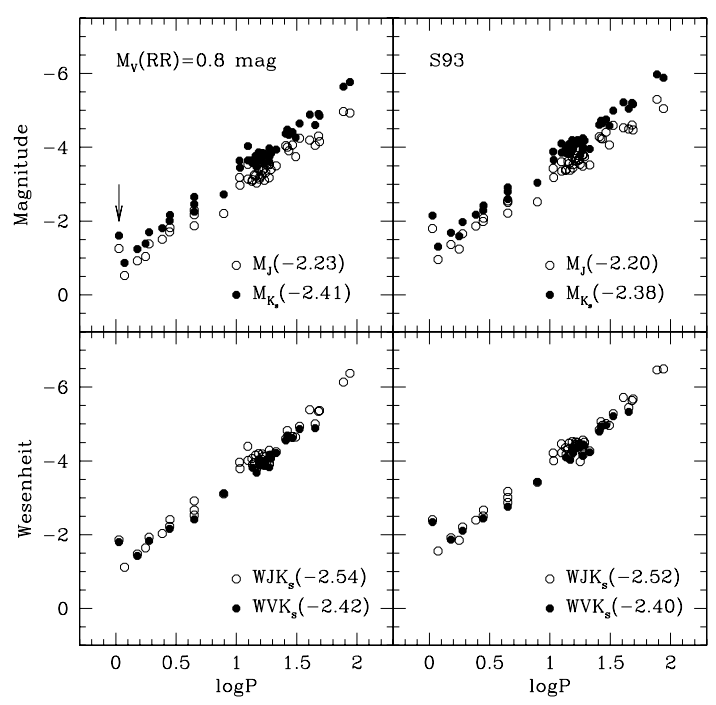

Fig. 5. $P M$ and $P W$ distributions of observed $\mathrm{P} 2 \mathrm{Cs}$ under two different assumptions on the absolute magnitude of RR Lyrae stars. The numbers in parentheses are the slopes of the relations, as derived by a linear regression to the data. The arrow indicates V7 in NGC 6341. The infrared magnitudes are taken from Matsunaga et al. (2006).

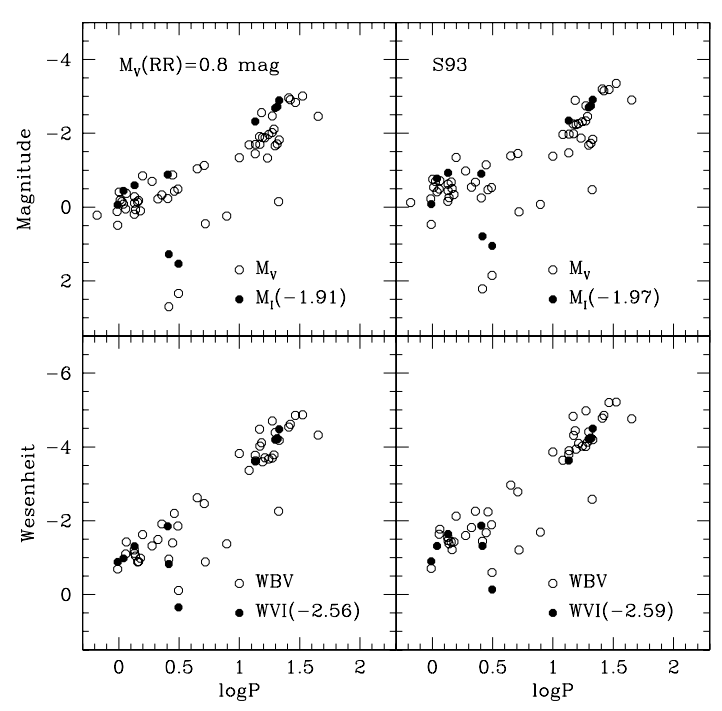

Fig. 6. $P M$ and $P W$ distributions of observed $P 2 C$ s under two different assumptions on the absolute magnitude of RR Lyrae stars. The outliers are V24 and V28 in NGC 4372, V12 and V32 in NGC 6205 and V3 in NGC 6254. The optical magnitudes are taken from Pritzl et al. (2003).

$\mu_{0, W B V}$ values are expected to be affected by large errors as a result of the older and less accurate photometry in these bands;

- for each given variable, the intrinsic distance modulus inferred by the near-infrared $W J K$ function is in a general agreement with the values based on the $W V J, W V H$ and $W V K$ functions. No sure comparison can unfortunately be made with $\mu_{0, W V I}$, whereas there is significant discrepancies with the results based on $W B V$. This can be due to some old $B V$ data (see also Pr03), as well as, in the case of $\omega$ Cen variables, to the occurrence of a metallicity spread;

- except the variables in $\omega$ Cen, the trend of the intrinsic and the apparent distance moduli, as determined by $J H K$ magnitudes, yields $A_{J} / E(B-V) \sim 0.85, A_{H} / E(B-V) \sim 0.54$ and $A_{K} / E(B-V) \sim 0.40$, in reasonable agreement with the extinction laws mentioned in Sect. 3.
Table 7. Mean intrinsic distance moduli (in magnitudes), as derived by the Wesenheit functions of BL Her and W Vir stars (the quantity $N$ is the number of averaged values).

\begin{tabular}{lccccc}
\hline \hline Name & $\mu_{0}(\mathrm{BLH})$ & $N$ & $\mu_{0}(\mathrm{WV})$ & $N$ & $\mu_{0}(\mathrm{RR})$ \\
\hline N2808 & $14.99 \pm 0.06$ & 1 & - & - & 14.90 \\
N5139 & $13.84 \pm 0.08$ & 4 & $13.78 \pm 0.16$ & 4 & 13.62 \\
N6254 & $13.39 \pm 0.20$ & 4 & $13.56 \pm 0.16$ & 2 & 13.23 \\
N6273 & $14.69 \pm 0.06$ & 1 & $14.77 \pm 0.06$ & 2 & 14.71 \\
N6284 & $15.77 \pm 0.06$ & 2 & - & - & 15.93 \\
N6293 & $14.80 \pm 0.06$ & 1 & - & - & 14.76 \\
N6402 & $14.80 \pm 0.08$ & 8 & $14.93 \pm 0.09$ & 8 & 14.86 \\
N6441 & $15.55 \pm 0.07$ & 2 & $15.53 \pm 0.19$ & 6 & 15.28 \\
N6441mp & $15.61 \pm 0.07$ & 2 & $15.58 \pm 0.18$ & 6 & 15.60 \\
N6715 & $17.19 \pm 0.10$ & 2 & - & - & 17.16 \\
N6749 & $14.56 \pm 0.08$ & 1 & - & - & 14.51 \\
N6779 & $15.05 \pm 0.06$ & 4 & $15.31 \pm 0.11$ & 4 & 15.08 \\
\hline
\end{tabular}

Eventually, by excluding the $\mu_{0, W B V}$ values, the mean intrinsic distance moduli listed in Table 7 together with the estimated total uncertainty. As a matter of comparison, we give in the last column the cluster distance determined by adopting the relation $M_{V}(\mathrm{RR})=0.89+0.22[\mathrm{Fe} / \mathrm{H}]$ and the reddening and $\mathrm{HB}$ visual magnitude in Table 6. As a whole, given the well known debate on the RR Lyrae distance scale (see the recent review by Cacciari \& Clementini 2003), we believe that the P2Cs distances agree with the RR Lyrae based values within the estimated uncertainty. As for a change in the $P M$ and $P W$ slopes around $\log P \sim 1$, namely between BL Her and W Vir stars, the values listed in Table 8 show similar distances for the variables in $\omega$ Cen and NGC 6441, whereas for the remaining clusters there is a subtle discrepancy as the former distance moduli are shorter by $\sim 0.15$ mag than the latter ones.

We can now study NGC $6341 \mathrm{~V} 7$ which has a period $\log P=$ 0.026 and is deviant from the near-infrared $P M$ relations (see arrow in Fig. 5). With $V J H K$ data taken from Del Principe et al. (2005), we would derive $\mu_{0}=13.80 \pm 0.07 \mathrm{mag}$ or $14.10 \pm 0.07 \mathrm{mag}$, depending on whether the variable is a fundamental or first-overtone BL Her star (i.e., adopting $\log P_{\mathrm{F}}=$ 0.038). As a whole, these distances are too short with respect to $\mu_{0}(R R)=14.65 \mathrm{mag}$, as derived by the relation $M_{V}(\mathrm{RR})=$ $0.89+0.22[\mathrm{Fe} / \mathrm{H}]$ using the values listed in Table 6 . On the other hand, comparing the $K$ magnitudes of RR Lyrae stars from Del Principe et al. (2005) with the predicted $P M_{K}$ relations presented by Del Principe et al. (2006), we derive $\mu_{0}(R R)=14.63 \pm$ 0.08 mag. Since the relations given in the present paper and those reported by Del Principe et al. (2006) are based on model computations which adopt homogeneous physics and numerical procedures, we can conclude that V7 is not a BL Her star but, due to the evidence that it is brighter than expected for its period, it may be an Anomalous Cepheid. Indeed, using for V7 the $P M_{K}$ relations determined by Fiorentino et al. (2006) from evolutionary and pulsation models of fundamental ACs, we derive $\mu_{K}(\mathrm{AC})=$ $14.69 \pm 0.15 \mathrm{mag}$.

\section{Conclusions}

The main results of this study can be summarized as follows:

- On the basis of updated nonlinear convective pulsation models of BL Herculis we derive analytical relations for the boundaries of the instability strip as a function of the adopted stellar physical parameters, as well as derive the pulsation equation for these bright objects. Moreover, we confirm earlier suggestions that for each given mass and helium content 
there exists an "intersection" luminosity, as given by the intersection between the FOBE and the FBE, above which only the fundamental mode is stable.

- By combining the pulsational results with the predictions of the evolutionary models by Pietrinferni et al. (2004, 2006), we select models brighter than the "intersection" luminosity and therefore pulsating only in the fundamental mode, corresponding to periods longer than $\sim 0.8 \mathrm{~d}$ and stellar masses $\leq 0.62 M_{\odot}$.

- For the models which have masses and luminosities consistent with the physical parameters adopted in the pulsation models, we derive the predicted $P M$ and $P W$ relations at the various photometric bands. We show that the predicted slopes are in close agreement with the empirical ones, quite independently of the slope in the adopted $M_{V}(\mathrm{RR})-[\mathrm{Fe} / \mathrm{H}]$ relation.

- The predicted $P M$ and $P W$ relations have been applied to all the known P2Cs in Galactic globular clusters and the resulting distance moduli are in statistical agreement with the RR Lyrae based values.

- The variables in $\omega$ Cen and NGC 6441 seem to support the hypothesis of unique $P M$ and $P W$ relations for BL Her and W Vir stars. Conversely, for the remaining clusters the former distance moduli are found to be shorter by $\sim 0.15 \mathrm{mag}$ than the latter ones, suggesting steeper relations with $P \geq$ 10 days. On this ground, no firm conclusion can presently be found in favor or against a change in the $P M$ and $P W$ slopes around $\log P \sim 1$.

- Finally, the application of the predicted relations to NGC 6341 V7 provides evidence that this variable is not a P2C. Using the results presented by Fiorentino et al. (2006), we confirm the earlier suggestion by Ma06 that this star can be the second AC in Galactic globular clusters.

Acknowledgements. Financial support for this study was provided by MIUR, under the scientific project "On the evolution of stellar systems: fundamental step toward the scientific exploitation of VST" (P.I. Massimo Capaccioli) and by INAF, under the scientific project "A laboratory for the theoretical study of stellar populations” (P.I. A. Buzzoni).

\section{References}

Bessell, M. S., \& Brett, J. M. 1988, PASP, 100, 1134B Bono, G., \& Stellingwerf, R. F. 1994, ApJS, 93, 233B

Bono, G., Castellani, V., \& Stellingwerf, R. F. 1995, ApJ, 445, 145

Bono, G., Caputo, F., \& Santolamazza, P. 1997, A\&A, 317, 171

Bono, G., Castellani, V., \& Marconi, M. 2000, ApJ, 532, L129
Bono, G., Castellani, V., \& Marconi, M. 2002, ApJ, 565, L83

Buchler, J. R., \& Moskalik, P. 1994, A\&A, 292, 450

Buchler, J. R., \& Buchler, N. E. G. 1994, A\&A, 285, 213

Cacciari, C., \& Clementini, G. 2003, in Stellar Candles for the Extragalactic Distance Scale, ed. D. Alloin, \& W. Gieren, Lect. Notes Phys., 635, 105

Caldwell, J. A. R., \& Coulson, I. M. 1987, AJ, 93, 1090

Cassisi, S., Salaris, M., \& Irwin, A. W. 2003, ApJ, 588, 862

Cassisi, S., Salaris, M., Castelli, F., \& Pietrinferni, A. 2004, ApJ, 616, 498

Castellani, V., Degl'Innocenti, S., \& Marconi 2002, A\&A, 387, 861

Caputo, F., Marconi, M., \& Musella, I. 2000, A\&A, 354, 610

Caputo, F., Castellani, V., Degl'Innocenti, S., Fiorentino, G., \& Marconi, M. 2004, A\&A, 424, 927C

Caputo, F., Bono, G., Fiorentino, G., Marconi, M., \& Musella, I. 2005, ApJ, 629, 1021

Cardelli, J. A., Clayton, G. C., \& Mathis, J. S. 1989, ApJ, 345, 245C

Carpenter, J. 2001, AJ, 121, 2851

Dean, J. F., Warren, P. R., \& Cousins, A. W. J. 1978, MNRAS, 183, 569

Del Principe, M., Piersimoni, A. M., Bono, G., et al. 2005, AJ, 129, 2714

Del Principe, M., Piersimoni, A. M., Storm, J., et al. 2006, ApJ, 652, 362

Di Criscienzo, M., Marconi, M., \& Caputo, F. 2004, ApJ, 612, 1092D

Di Criscienzo, M., Caputo, F., Marconi, M., \& Musella, I. 2006, MNRAS, 365, 1357

Dorman, B., Rood, R. T., \& O’ Connel, R. W. 1993, 419, 596

Fiorentino, G., Limongi, M., Caputo, F., \& Marconi, M. 2006, A\&A, 460, 155

Fiorentino, G., Marconi, M., Musella, I., Caputo, F. 2007, A\&A, in press [arXiv: astro-ph/0707.0959v1]

Gingold, R. A. 1985, MmSAI, 56, 169

Harris, H. C. 1985 , AJ, 90, 756

Harris, H. C. 1996, AJ, 112, 1487

Kubiak, M., \& Udalski, A. 2003, AcA, 53, 117

Laney, C., \& Stobie, R. S. 1993, MNRAS, 263, L921

Madore, B. F. 1982, ApJ, 253, 57

Madore, B. F., \& Freedman, W. L. 1991, PASP, 103, 933

Marconi, M., \& Di Criscienzo, M. 2007, A\&A, in press [arXiv:astro-ph/0701256]

Marconi, M., Caputo, F., Di Criscienzo, M., \& Castellani, M. 2003, ApJ, 596, 299M

Marconi, M., Fiorentino, G., \& Caputo, F. 2004, A\&A, 417, 1101

Marconi, M., Musella, I., \& Fiorentino, G. 2005, ApJ, 632, 590

Matsunaga, N., Fukushi, H., Nakada, Y., et al. 2006, MNRAS, 370, 1979

McNamara, D. H. 1995, AJ, 109, 2134

Nemec, J. M., Nemec, A. F. L., \& Lutz, T. E. 1994, AJ, 108, 222

Pritzl, B., Smith, H., Stetson, P., et al. 2003, AJ, 126, 1381

Pietrinferni, A., Cassisi, S., Salaris, M., \& Castelli, F. 2004, ApJ, 612, 168

Pietrinferni, A., Cassisi, S., Salaris, M., \& Castelli, F. 2006, ApJ, 642, 797

Recio-Blanco, A., Piotto, G., de Angeli, F., et al. 2005, A\&A, 432, 851R

Riello, M., Cassisi, S., Piotto, G., et al. 2003, A\&A, 410, 553

Sandage, A., \& Tammann, G. 2006, ARA\&A, 44, 93

Salaris, M., Riello, M., Cassisi, S., \& Piotto, G. 2004, A\&A, 420, 911 S

Sandage, A. 1993, AJ, 106, 687S

Stellingwerf, R. F. 1982, ApJ, 262, 330S

Tuggle, R., \& Iben, I. 1972, ApJ, 8, 5

Wallerstein, G. 1990, ASPC, 11, 56

Wallerstein, G. 2002, PASP, 114, 689

Wallerstein, G., \& Cox, A. N. 1984, PASP, 96, 677 